Supporting information

\title{
Theoretical Investigation of Propylene Epoxidation on Ag(111) by Molecular Oxygen: $\mathrm{Na}(\mathrm{K}, \mathrm{Cl})$ Effects
}

\begin{abstract}
Bo Zhao, Gui-Chang Wang*
(Key Laboratory of Advanced Energy Materials Chemistry (Ministry of Education) and the Tianjin key Lab and Molecule-based Material Chemistry, College of Chemistry, Nankai University, Tianjin 300071, P. R. China)
\end{abstract}

*Corresponding author: Gui-Chang Wang. E-mail: wangguichang@nankai.edu.cn

Telephone: +86-22-23503824(O) Fax: +86-22-23502458 
Table S1 Adsorption energies of $\mathrm{Na}(\mathrm{K}, \mathrm{Cl})$ on $\mathrm{Ag}(111)$ (unit: eV)

\begin{tabular}{cccc}
\hline Adsorption sites & $\mathrm{Na}$ & $\mathrm{K}$ & $\mathrm{Cl}$ \\
Top & -1.65 & -1.92 & - \\
Bridge & -1.76 & -1.96 & -3.11 \\
Hcp & -1.77 & -1.97 & -3.18 \\
Fcc & 1.78 & -1.97 & -3.19 \\
\hline
\end{tabular}

Note: $\mathrm{Cl}$ atom can not stable at Top site and moves to Fcc site.

Table S2 Energetic results of each elemental step of propylene epoxidation (unit: eV)

\begin{tabular}{|c|c|c|c|c|c|c|c|c|}
\hline & \multicolumn{2}{|c|}{$\operatorname{Ag}(111)$} & \multicolumn{2}{|c|}{$\mathrm{Na} / \mathrm{Ag}(111)$} & \multicolumn{2}{|c|}{$\mathrm{K} / \operatorname{Ag}(111)$} & \multicolumn{2}{|c|}{$\mathrm{Cl} / \mathrm{Ag}(111)$} \\
\hline & $\underline{\Delta E}$ & $E_{a}$ & $\underline{\Delta E}$ & $\underline{E}_{a}$ & $\underline{\Delta E}$ & $\underline{E}_{a}$ & $\underline{\Delta E}$ & $\underline{E}_{a}$ \\
\hline $\mathrm{C}_{3} \mathrm{H}_{6} *+\mathrm{O}_{2} * \rightarrow \mathrm{C}_{3} \mathrm{H}_{5}{ }^{*}+\mathrm{OOH}^{*}$ & 0.19 & 0.47 & 0.24 & 0.50 & 0.27 & 0.52 & 0.29 & 0.71 \\
\hline $\mathrm{C}_{3} \mathrm{H}_{6} *+\mathrm{O}_{2} * \rightarrow \mathrm{OOMMP}^{*}+*$ & -0.06 & 0.19 & -0.07 & 0.22 & -0.06 & 0.24 & -0.07 & 0.17 \\
\hline $\mathrm{OOMMP} 2 *+* \rightarrow \mathrm{OMMP} 2 *+\mathrm{O} *$ & -1.10 & 0.31 & -1.81 & 0.34 & -1.70 & 0.39 & -0.81 & 0.30 \\
\hline $\mathrm{OMMP} 2 * \rightarrow \mathrm{PO}+*$ & -0.51 & 0.62 & -0.59 & 0.63 & -0.54 & 0.74 & -0.87 & 0.51 \\
\hline $\mathrm{OMMP} 2 * \rightarrow \mathrm{AC}+*$ & -1.66 & 0.68 & -1.79 & 0.92 & -1.78 & 0.95 & -1.98 & 0.67 \\
\hline
\end{tabular}

Table S3 Net Bader charge of two-sites-four-center models on $\mathrm{Ag}(111), \mathrm{K}(\mathrm{Cl}) / \mathrm{Ag}(111)$ for AHS route(unit: |e|)

\begin{tabular}{cccc}
\hline & $\operatorname{Ag}(111)$ & $\mathrm{K} / \mathrm{Ag}(111)$ & $\mathrm{Cl} / \mathrm{Ag}(111)$ \\
$\mathrm{O}$ & -0.51 & -0.61 & -0.51 \\
$\mathrm{H}$ & +0.39 & +0.40 & +0.40 \\
$\mathrm{C}_{3} \mathrm{H}_{5}$ & +0.18 & +0.27 & +0.15 \\
$\mathrm{Ag}$ & +0.10 & +0.10 & +0.10 \\
\hline
\end{tabular}


$d_{N a-d \varepsilon}=3.05 / 3.05 / 3.06 \AA, E_{a d s}=-1.78 \mathrm{eV}$

0
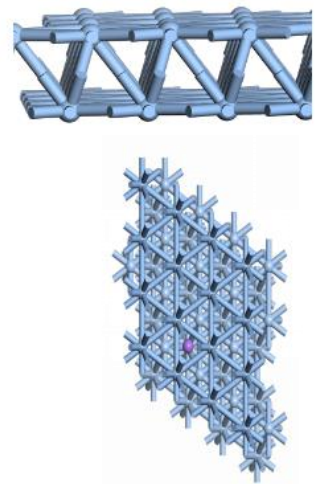

$d_{K-A \varepsilon}=3.40 / 3.40 / 3.41 \AA, E_{a d s}=-1.97 \mathrm{eV}$

C
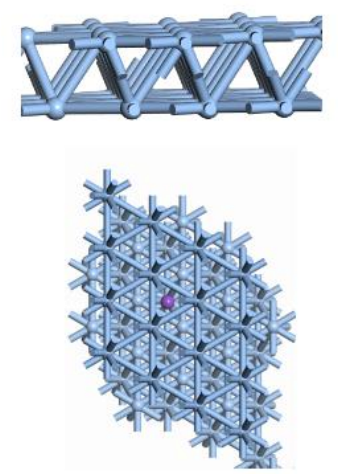

$d_{C I-d \varepsilon}=2.65 / 2.64 / 2.65 \AA, E_{a d s}=-3.19 \mathrm{eV}$
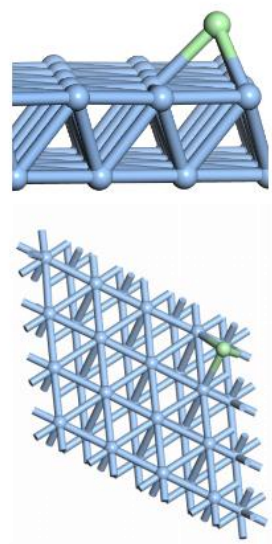

Fig. S1 Side and top views of most stable adsorption configuration of $\mathrm{Na}, \mathrm{K}$ and $\mathrm{Cl}$ on $\mathrm{Ag}(111)$
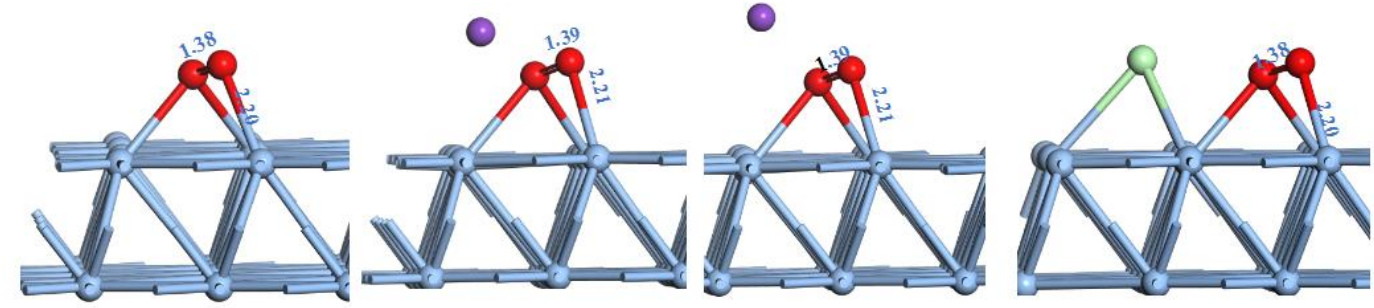

Fig.S2 Molecular oxygen adsorption configuration on $\mathrm{Ag}(111), \mathrm{Na} / \mathrm{Ag}(111), \mathrm{K} / \mathrm{Ag}(111)$ and $\mathrm{Cl} / \mathrm{Ag}(111)$ faces(unit: $\AA)$ 

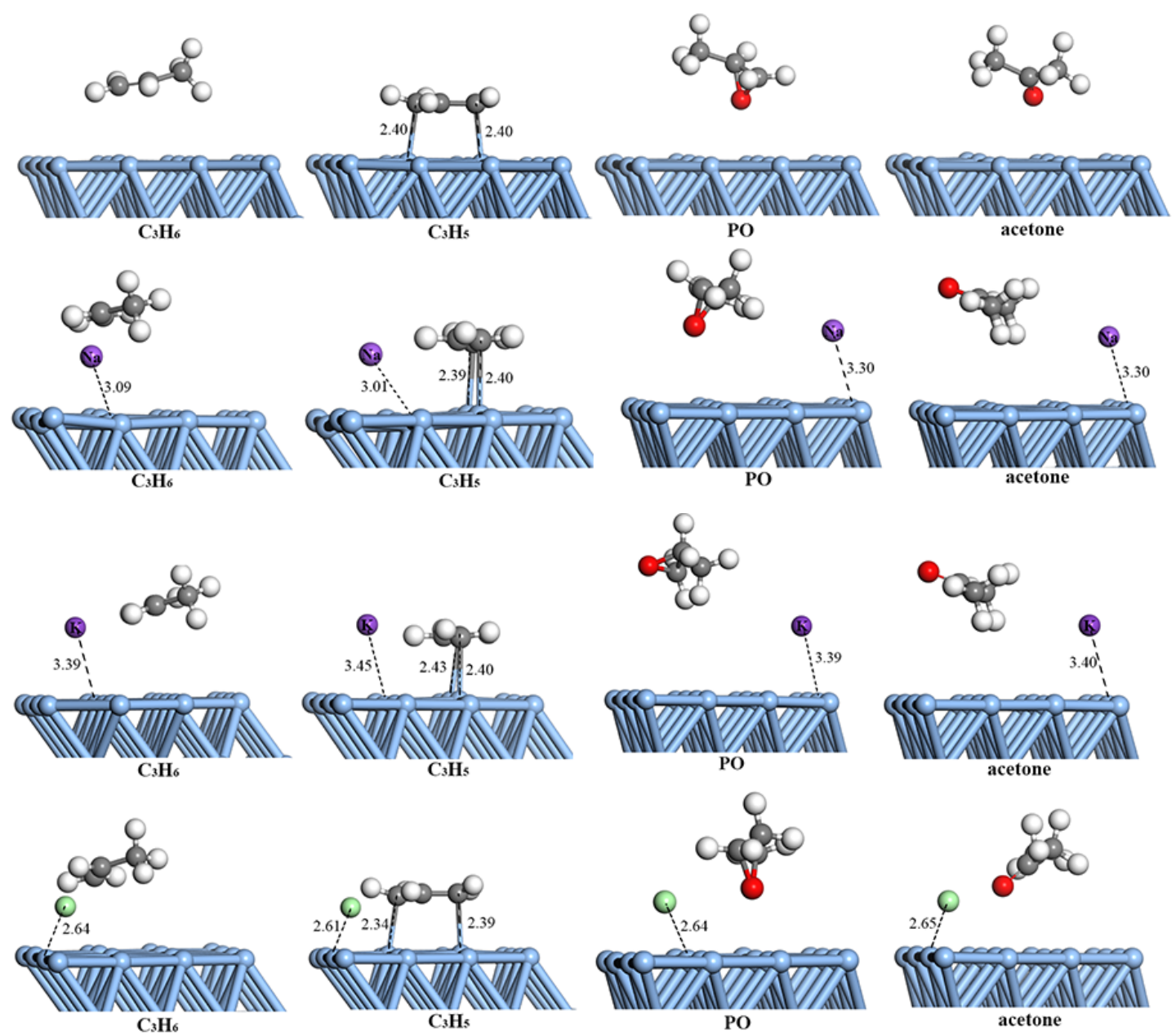

Fig. S3 Possible adsorption configuration of key species of propylene epoxidation on clean, $\mathrm{Na}, \mathrm{K}$, and $\mathrm{Cl}$ modified $\operatorname{Ag}(111)$ faces(unit: $\AA$ )
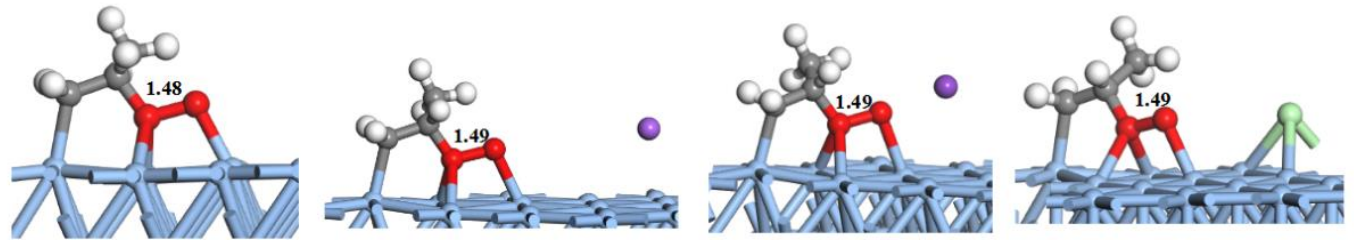

Fig.S4 OOMMP2 configuration on $\mathrm{Ag}(111), \mathrm{Na} / \mathrm{Ag}(111), \mathrm{K} / \mathrm{Ag}(111)$ and $\mathrm{Cl} / \mathrm{Ag}(111)$ faces(unit: $\AA$ ) 

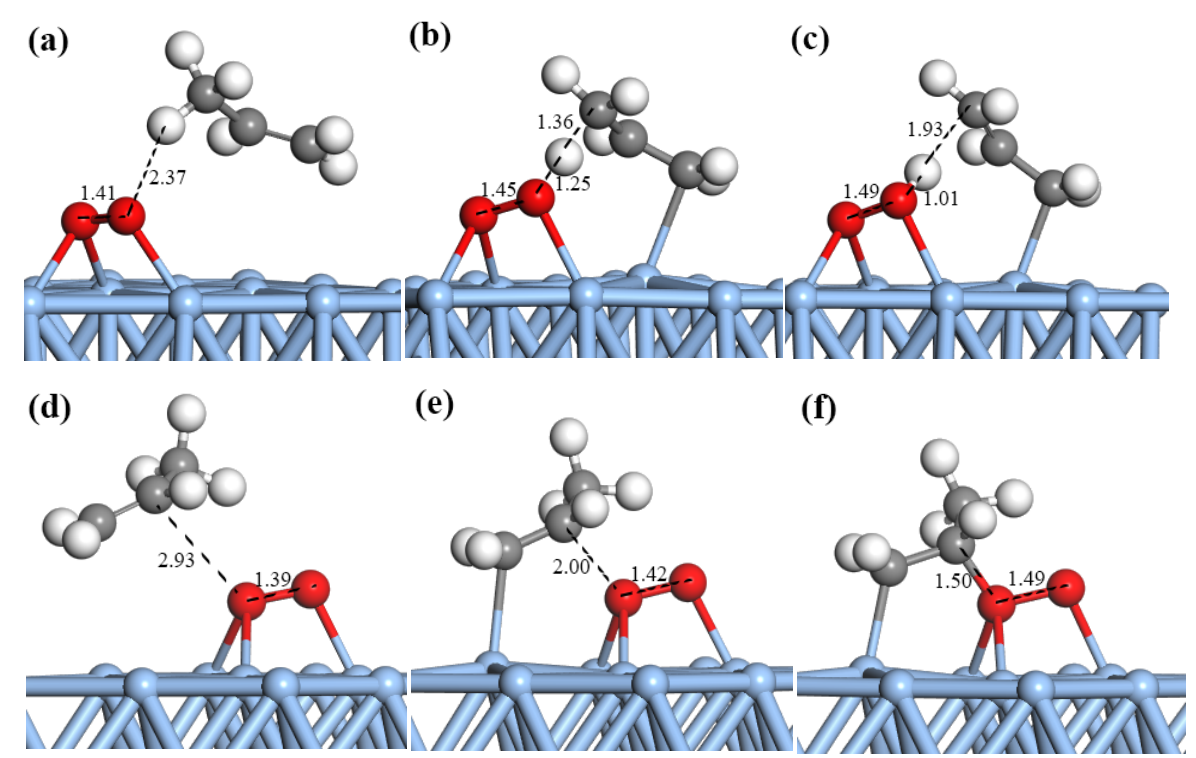

Fig.S5 Reaction networks of propylene reaction on $\operatorname{Ag}(111)$. (a) , (b) and (c) mean the IS, TS and FS of AHS step, and (d), (e), and (f) mean the IS, TS, FS of OOMMP2 formation step (unit in $\AA$ ). 

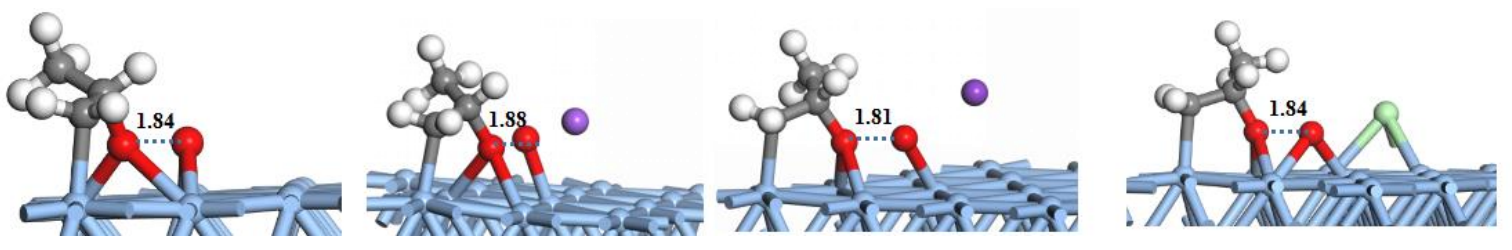

Fig.S6 TSs of OOMMP2 $\rightarrow \mathrm{OMMP} 2+\mathrm{O}$ on pure and $\mathrm{Na}(\mathrm{K}, \mathrm{Cl})$ modified $\mathrm{Ag}(111)$ faces

(a)

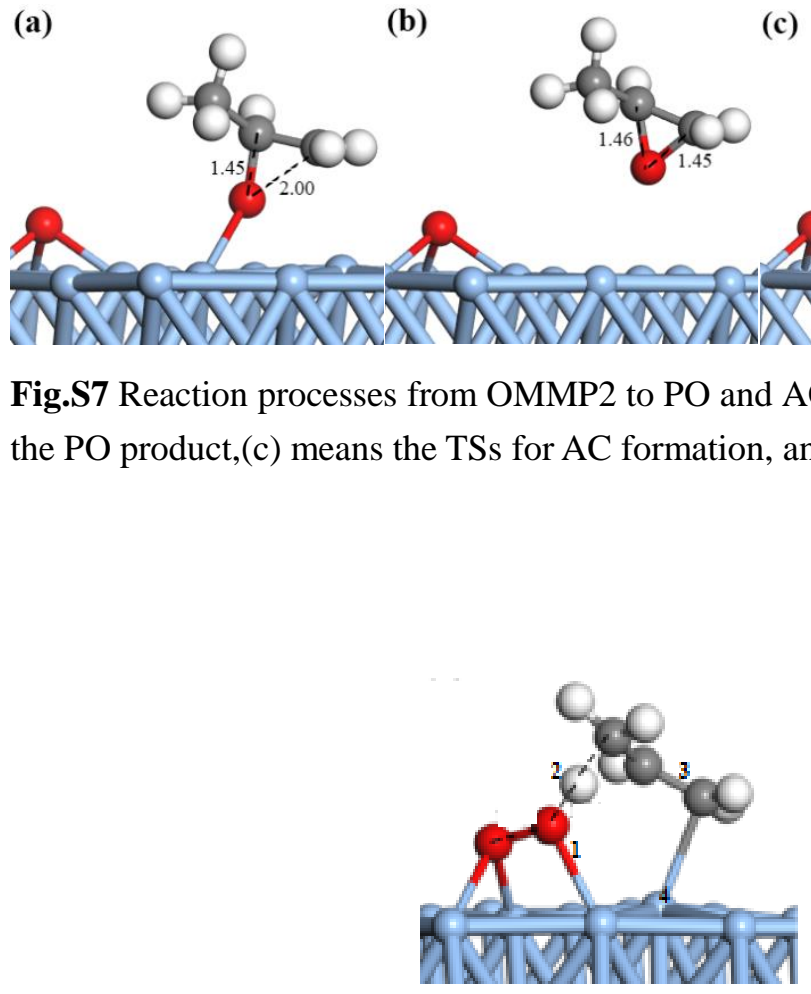

(c)

(d)

Fig. S8 Two-site-four-center model of AHS on $\mathrm{Ag}(111), \mathrm{K} / \mathrm{Ag}(111)$ and $\mathrm{Cl} / \mathrm{Ag}(111)$ 